\title{
FITASE EM DIETAS PARA SUÍNOS EM CRESCIMENTO: (I) IMPACTO AMBIENTAL ${ }^{1}$
}

\author{
PHYTASE IN DIETS FOR GROWING PIGS: \\ (I) ENVIRONMENTAL IMPACT
}

\section{Maria do Carmo Mohaupt Marques Ludke ${ }^{2}$ Jorge López $^{3}$ Jorge Vitor Ludke $^{4}$}

\section{RESUMO}

O objetivo deste trabalho foi determinar o nível adequado de fitase (0,300, 600 e 900UF/kg da dieta) a ser adicionado em dietas de suínos que continha dois níveis de proteína bruta (PB), um na exigência do animal (18\%; NRC, 1988) e outro em nível menor (16\%), para a redução do nitrogênio, fósforo e cálcio excretados, com o intuito de evitar poluição ambiental. Foram utilizados 24 leitões com peso médio inicial de 18,4kg alojados em gaiolas metabólicas para ensaio de metabolismo através do método de coleta total usando o marcador óxido férrico. Dietas isoenergéticas com $3295 \mathrm{kcal}$ de EM/kg foram fornecidas para os animais de acordo com o peso metabólico. Os parâmetros avaliados foram: quantidade de nitrogênio consumido e excretado total; quantidade de fósforo consumido e excretado nas fezes e urina; quantidade de cálcio consumido e excretado nas fezes e urina. Foi observado um aumento na biodisponibilidade dos nutrientes avaliados ao ser adicionado fitase nos níveis entre 421 - 466UF/kg da dieta.

Palavras-chave: cálcio, enzima, excreção, fitato, fósforo, nitrogênio

\section{SUMMARY}

The objective of this experiment was determine the appropriate level of phytase (0,300,600 and 900PU/kg of the diet) to be added in swine diets containing two levels of crude protein (CP), one in the demand of the animal (18\% - NRC, 1988) and another in lower level (16\%), for evaluation of the most effective reduction in excretion of nitrogen, phosphorus and calcium, to avoid larger environmental problems. Twenty four pigs with an initial avearage weight of $18.4 \mathrm{~kg}$ were housed in metabolic cages for determination of the nitrogen, phosphorus and calcium metabolism through the method of total colection of faeces using ferric oxide as a marker. The diets were supplied for the animals related to their metabolic weight, and were isoenergetic with $3295 \mathrm{kcal}$ of $\mathrm{ME} / \mathrm{kg}$. The parameters evaluated were the amount of consumed and total excreted nitrogen; amount of phosphorus and calcium consumed and excreted in the feces and in the urine. An increase in the biodisponibility of these nutrients was observed when the phytase was added in the levels between 421 - 466PU/kg of the diet.

Key words: calcium, enzyme, excretion, nitrogen, phosphorus, , phytate

\section{INTRODUÇÃO}

A maioria do fósforo nos grãos de cereais está na forma do complexo orgânico fitato. Devido ao grupo ortofosfato do fitato ser altamente ionizado, este complexa com uma variedade de cátions ( $\mathrm{Ca}, \mathrm{Fe}, \mathrm{Zn}, \mathrm{Mn}$, etc) e grupo amina de alguns aminoácidos básicos (LEHNINGER et al. 1993). Esse complexo caracteriza o fitato como um fator anti-nutricional por diminuir a disponibilidade

\footnotetext{
${ }^{1}$ Parte da Tese de Doutorado apresentada pela primeiro autor à Faculdade de Agronomia da Universidade Federal do Rio Grande do Sul (UFRGS).

${ }^{2}$ Zootecnista, Doutor, bolsista Recém-Doutor do CNPq no CNPSA - EMBRAPA. Rua Independência, 283, apt. 304/A, 89700-000,

Concórdia, SC. E-mail: jmludke@ netcon.com.br. Autor para correspondência.

${ }^{3}$ Professor Titular do Departamento de Zootecnia da Faculdade de Agronomia da UFRGS.

${ }^{4}$ Pesquisador de Nutrição de Suínos na EMBRAPA - Suínos e Aves. Concórdia - SC. 
de minerais e proteínas (O'DELL \& DeBOLLAND, 1976). Com isso, o fósforo fítico, por ser de baixa disponibilidade para os não-ruminantes, juntamente com o excesso de fosfato inorgânico adicionado às rações é eliminado nas fezes dos animais. Isso é motivo de um alto índice de poluição ambiental em áreas de alta concentração de suínos e aves, como já vem ocorrendo na Holanda, devido a uma grande quantidade não apenas de fósforo, mas também de nitrogênio excretados pelos animais. Dentre as fases de criação dos suínos, são as fases de crescimento e terminação que são responsáveis pela maior quantidade de excreção do nitrogênio, pois cerca de $2 / 3$ do nitrogênio excretado pelos animais no sistema de produção ocorre nestas fases (PINNOT, 1990).

O potencial do fósforo para contaminar a terra e a água é menor que do nitrogênio, em função do fósforo aderir às partículas do solo, tornando-se assim, um contaminante parcial de rios e lagos, devido à sua translocação limitada (COFFEY, 1992). No entanto, em contato com a superfície das águas, o fosfato estimula o crescimento das algas, processo chamado de eutroficação, resultando em um decréscimo na qualidade desta água. A morte e deteriorização destas algas diminui a quantidade de oxigênio na água, criando um meio inadequado para os peixes e outros animais aquáticos (CROMWELL et al., 1995).

O nitrogênio possui uma translocação maior quando comparado com o fósforo, ao entrar em contato com o solo, sendo transformado em nitrato, nitrito e amônia (nitrificação), que representam as principais substâncias poluentes das águas e também do ar (ENG, 1996). Segundo STILBORN (1998), existem diferentes estratégias para a redução na excreção do fósforo e nitrogênio pelos não-ruminantes. Uma delas é suplementar as dietas com a enzima fitase.

O objetivo deste trabalho foi avaliar o nível adequado de fitase a ser adicionado em dietas para suínos, compostas por milho e farelo de soja, para reduzir a quantidade de nitrogênio, fósforo e cálcio excretados, evitando maiores problemas ambientais. Outro objetivo foi verificar em qual dos níveis de proteína (16\% e/ou 18\% PB) a enzima tem maior eficácia.

\section{MATERIAL E MÉTODOS}

O experimento foi realizado na sala de metabolismo, climatizada com temperatura média de $18,5^{\circ} \mathrm{C}$, do Centro Nacional de Pesquisa de Suínos e Aves (CNPSA) - EMBRAPA localizada em Concórdia - SC, Brasil, no período de 01 de junho a 28 de junho de 1996. Foram utilizados 24 leitões machos castrados F2, provenientes da cruza de fêmeas F1 (Landrace X Large White) e macho sintético M558 (Pietran X Duroc X Hampshire), com peso inicial e final médio de 18,4 e $32,3 \mathrm{~kg}$, respectivamente. Todos receberam água à vontade.

Os animais foram submetidos a ensaios de digestibilidade aparente, com 4 dias de coleta de fezes e urina/semana em 4 períodos (semana) de avaliação. Os leitões foram pesados semanalmente e foi utilizado o óxido férrico na ração em concentração de $0,125 \%$ como marcador. Foi avaliada a quantidade de nitrogênio (QNC), quantidade de fósforo (QPC) e quantidade de cálcio $(\mathrm{QCaC})$, consumidos através da dieta.

Para obtenção da quantidade de nitrogênio excretado total (QNET) foi analisada a concentração deste nutriente nas fezes e urina, de acordo com o método descrito na AOAC (1984). As quantidades de fósforo e cálcio excretados nas fezes (QPF e QCaF, respectivamente) também foram avaliados por este método. No entanto, as quantidades de fósforo e cálcio excretados na urina (QPU e QCaU, respectivamente) foram determinadas segundo o método do LABTEST (1985) para fósforo e LABTEST (1996) para cálcio.

As dietas foram fornecidas aos animais de acordo com seu peso metabólico (PM). Esses receberam ao dia 3 vezes a energia de mantença, considerada $110 \mathrm{kcal} / \mathrm{kg} / \mathrm{dia}$ (NRC, 1988). Foram dietas isoenergéticas com $3295 \mathrm{kcal}$ de $\mathrm{EM} / \mathrm{kg}$, com dois níveis de Proteína bruta (PB) $18 \%$ (NRC, 1988) e $16 \%$ (abaixo da exigência do animal), e quatro níveis de fitase: $0,300,600$ e $900 \mathrm{UF} / \mathrm{kg}$ da dieta. A composição das dietas com a respectiva análise químico-bromatológica ou calculada é mostrada na tabela 1. Ao ser adicionada a fitase na dieta, foi reduzida a concentração do suplemento inorgânico, de acordo com a BASF (1997). Esta enzima utilizada é de nome comercial NATUPHOS 5.000, que é fornecida pela BASF. O teor de cálcio das dietas com fitase foi reduzido em $10 \%$ em relação à concentração de cálcio da dieta testemunha (sem fitase e com fosfato inorgânico), ficando as dietas suplementadas com os diferentes níveis de fitase $(300,600$ e $900 \mathrm{UF} / \mathrm{kg})$ com a mesma concentração de cálcio. 


\begin{tabular}{|c|c|c|c|c|c|c|c|c|}
\hline \multirow[t]{3}{*}{ Ingrediente (\%) } & \multicolumn{8}{|c|}{ Tratamento } \\
\hline & \multicolumn{4}{|c|}{$16 \% \mathrm{~PB}$} & \multicolumn{4}{|c|}{$18 \% \mathrm{~PB}$} \\
\hline & 0 & 300 & 600 & 900 & 0 & 300 & 600 & 900 \\
\hline Milho & 72,80 & 72,80 & 72,80 & 72,80 & 67,74 & 67,74 & 67,74 & 67,74 \\
\hline F. de Soja & 21,51 & 21,51 & 21,51 & 21,51 & 27,02 & 27,02 & 27,02 & 27,02 \\
\hline Öleo de Soja & 1,56 & 1,56 & 1,56 & 1,56 & 1,35 & 1,35 & 1,35 & 1,35 \\
\hline Fosfato Bicálcico & 1,41 & 1,02 & 0,63 & 0,23 & 1,37 & 0,98 & 0,59 & 0,20 \\
\hline Calcáreo & 0,84 & 0,91 & 1,18 & 1,45 & 0,83 & 0,89 & 1,16 & 1,43 \\
\hline L-Lisina & 0,21 & 0,21 & 0,21 & 0,21 & 0,02 & 0,02 & 0,02 & 0,02 \\
\hline Premix Vitamínico ${ }^{1}$ & 0,30 & 0,30 & 0,30 & 0,30 & 0,30 & 0,30 & 0,30 & 0,30 \\
\hline Cloreto de Colina & 0,22 & 0,22 & 0,22 & 0,22 & 0,22 & 0,22 & 0,22 & 0,22 \\
\hline Premix mineral $^{2}$ & 0,30 & 0,30 & 0,30 & 0,30 & 0,30 & 0,30 & 0,30 & 0,30 \\
\hline Sal & 0,35 & 0,35 & 0,35 & 0,35 & 0,35 & 0,35 & 0,35 & 0,35 \\
\hline Aditivo e promotores ${ }^{3}$ & 0,50 & 0,50 & 0,50 & 0,50 & 0,50 & 0,50 & 0,50 & 0,50 \\
\hline Caulim & 0 & 0,26 & 0,32 & 0,39 & 0 & 0,27 & 0,33 & 0,39 \\
\hline Fitase & 0 & 0,06 & 0,12 & 0,18 & 0 & 0,06 & 0,12 & 0,18 \\
\hline Total & 100 & 100 & 100 & 100 & 100 & 100 & 100 & 100 \\
\hline \multicolumn{9}{|l|}{ Nutrientes } \\
\hline MS,$\%^{4}$ & 87,12 & 87,09 & 87,01 & 87,04 & 87,05 & 86,99 & 86,98 & 87,01 \\
\hline $\mathrm{EE}, \%^{4}$ & 4,37 & 4,49 & 4,65 & 4,46 & 4,30 & 4,21 & 4,25 & 4,21 \\
\hline EM $(\mathrm{kcal} / \mathrm{kg})^{5}$ & 3295 & 3295 & 3295 & 3295 & 3295 & 3295 & 3295 & 3295 \\
\hline $\mathrm{PB}, \%^{4}$ & 16,32 & 16,45 & 16,30 & 16,16 & 18,57 & 18,41 & 18,08 & 18,65 \\
\hline $\mathrm{PB}, \%^{5}$ & 16,00 & 16,00 & 16,00 & 16,00 & 18,00 & 18,00 & 18,00 & 18,00 \\
\hline Lisina, $\%^{5}$ & 0,95 & 0,95 & 0,95 & 0,95 & 0,95 & 0,95 & 0,95 & 0,95 \\
\hline $\mathrm{FB}, \%^{4}$ & 2,52 & 2,59 & 2,52 & 2,53 & 2,67 & 2,62 & 2,86 & 2,62 \\
\hline Cinzas, $\%^{4}$ & 4,21 & 4,33 & 3,98 & 4,10 & 4,38 & 4,31 & 4,42 & 4,33 \\
\hline Cálcio, $\%{ }^{4}$ & 0,54 & 0,57 & 0,49 & 0,56 & 0,63 & 0,50 & 0,56 & 0,54 \\
\hline Cálcio, $\%^{5}$ & 0,70 & 0,63 & 0,63 & 0,63 & 0,70 & 0,63 & 0,63 & 0,63 \\
\hline P Total, $\%^{4}$ & 0,51 & 0,45 & 0,39 & 0,36 & 0,55 & 0,45 & 0,38 & 0,38 \\
\hline P Total, $\%^{5}$ & 0,57 & 0,50 & 0,43 & 0,36 & 0,58 & 0,51 & 0,44 & 0,38 \\
\hline $\mathrm{P}$ disponível,$\%^{5}$ & 0,35 & 0,28 & 0,21 & 0,14 & 0,35 & 0,28 & 0,21 & 0,14 \\
\hline Relação Ca:P ${ }^{4}$ & $1,1: 1$ & $1,3: 1$ & $1,3: 1$ & $1,6: 1$ & $1,1: 1$ & $1,1: 1$ & $1,5: 1$ & $1,4: 1$ \\
\hline Relação Ca:P $\mathrm{P}^{5}$ & $1,2: 1$ & $1,3: 1$ & $1,5: 1$ & $1,7: 1$ & $1,2: 1$ & $1,2: 1$ & $1,4: 1$ & $1,6: 1$ \\
\hline
\end{tabular}

${ }^{1}$ Premix vitamínico por kg de dieta: $11.250 \mathrm{UI}$ vit. A; $4.350 \mathrm{UI}$ vit. $\mathrm{D}_{3} ; 164,55 \mathrm{mg}$ vit. E; $11,22 \mathrm{mg}$ vit. K; $3,57 \mathrm{mg}$ vit. $\mathrm{B}_{1}, 9,9 \mathrm{mg}$ vit. $\mathrm{B}_{2} ; 3,84 \mathrm{mg}$ vit. $\mathrm{B}_{6}, 33,75 \mu \mathrm{c}$ vit. $\mathrm{B}_{12} ; 31,35 \mathrm{mg}$ ác. pantotênico; $57,15 \mathrm{mg}$ niacina; $0,36 \mathrm{mg}$ biotina; $1,89 \mathrm{mg}$ ácido fólico.) (Vitamin premix per $\mathrm{kg}$ of diet: : $11.250 \mathrm{UI}$ vit. A; 4,350 UI vit. $D_{3} ; 164,55 \mathrm{mg}$ vit. E; $11,22 \mathrm{mg}$ vit. $K ; 3,57 \mathrm{mg}$ vit. $\mathrm{B}_{1} ; 9,9 \mathrm{mg}$ vit. $\mathrm{B}_{2}, 3,84$ vit. $\mathrm{B}_{6} ; 33,75 \mathrm{mg}$ vit. $\mathrm{B}_{12} ; 31,35 \mathrm{mg}$ pantothenic acid; $57,15 \mathrm{mg}$ niacin; $0,36 \mathrm{mg}$ biotin; 1,89 folic acid).

${ }^{2}$ Premix mineral por kg de dieta: $199,8 \mathrm{mg} \mathrm{Fe} ; 249,5 \mathrm{mg} \mathrm{Cu} ; 61,2 \mathrm{mg} \mathrm{Mn} ; 153,9 \mathrm{mg} \mathrm{Zn} ; 1,72 \mathrm{mg}$ I. (Mineral premix per kg of diet: $199,8 \mathrm{mg} \mathrm{Fe} ; 249,5 \mathrm{mg} \mathrm{Cu} ; 61,2 \mathrm{mg} \mathrm{Mn} ; 153,9 \mathrm{mg} \mathrm{Zn} ; 1,72 \mathrm{mg} \mathrm{I})$

${ }^{3}$ Aditivos por kg de dieta: BHT 0,005\%; Promotor-leitão 0,5\% (91\% farelo de soja; 5\% TM100; $2 \%$ sulfametazina, $2 \%$ Olaquindox. (Aditive per kg of diet: 0,005 BHT; Pig Promotor 0,5\% (91\% soybean meal; 5\% TM100; $2 \%$ sulphametazin; 2\% Olaquindox).

${ }^{4}$ Valores determinados em laboratório (determined values in the laboratory)

${ }^{5}$ Valores Calculados (Calculated Values)

variância dos dados adotou-se o modelo estatístico representado por: $\mathrm{y}_{\mathrm{jik}}=\mu+\mathrm{b}_{\mathrm{j}}+\mathrm{p}_{\mathrm{i}}+$ $\mathrm{f}_{\mathrm{k}}+\mathrm{pf}_{\mathrm{ik}}+\mathrm{e}_{\mathrm{jik}}$; onde: $\mathrm{Y}_{\mathrm{jik}}$ é o valor observado da variável resposta que pertence a gaiola jik; $\mu=$ estimativa da média geral; $b_{j}=$ efeito do bloco $\mathrm{j} ; \mathrm{p}_{\mathrm{i}}=$ efeito do nível de proteína $\mathrm{i} ; \mathrm{f}_{\mathrm{k}}=$ efeito do nível de fitase $\mathrm{k} ; \mathrm{pf}_{\mathrm{ik}}=$ efeito $\mathrm{da}$ interação entre o nível de proteína i e o nível de fitase $\mathrm{k} ; \mathrm{e}_{\mathrm{jik}}=$ erro experimental. Realizouse o desdobramento da interação proteína $\mathrm{x}$ fitase, avaliando-se os níveis de fitase dentro de cada nível de proteína. O efeito dos níveis de fitase foi desdobrado em componentes linear, quadrática e cúbica ou modelo descontínuo linear platô $(\mathrm{y}=\mathrm{a}+\mathrm{b} *$ f, $\forall \mathrm{f} \leq \mathrm{f}^{*}$ e $\mathrm{y}=\mathrm{K} \forall \mathrm{f}$ $\left.\geq \mathrm{f}^{*} \operatorname{com} \mathrm{f}^{*}=(\mathrm{k}-\mathrm{a}) / \mathrm{b}\right)$. Os dados foram analisados segundo SAS (1990).

\section{RESULTADOS E DISCUSSÃO}

De acordo

$\mathrm{O}$ delineamento experimental foi em blocos casualizados com três blocos e 8 tratamentos, consistindo num fatorial $2 \times 4$, dois níveis de proteína bruta $(18 \%$ e $16 \%)$ e 4 níveis de fitase $(0$, 300, 600 e 900UF/kg da dieta). Cada repetição foi considerada uma unidade experimental representada por uma gaiola metabólica com um animal, e o critério de bloqueamento foi o peso dos animais no início do experimento. com o teste $\mathrm{F}$, foi encontrada interação proteína $\mathrm{x}$ fitase $(\mathrm{P}<0,001)$ sobre QPC e QCaC. Estes resultados são mostrados na tabela 2. No nível de $16 \%$ de $\mathrm{PB}$, observou-se um efeito linear $(\mathrm{P}<0,001)$ ao avaliar a $\mathrm{QPC}$, e efeito cúbico $(\mathrm{P}<0,001)$ ao avaliar a QCaC. No nível de $18 \%$ de $\mathrm{PB}$, as respectivas variáveis apresentaram um efeito linear platô. As equações são mostradas nas figuras 1 e 2 . 
Tabela 2 - Médias ajustadas e erro padrão da média da quantidade de nitrogênio consumido (QNC - $\left.\mathrm{g} / \mathrm{kg}^{0,75} \mathrm{xd}\right)$, quantidade de fósforo consumido (QPC - $\mathrm{g} / \mathrm{kg}^{0,75} \mathrm{xd}$ ) e quantidade de cálcio consumido $\left(\mathrm{QCaC}-\mathrm{g} / \mathrm{kg}^{0,75} \mathrm{xd}\right)$ em base de matéria seca e em relação ao peso metabólico dos animais.

\begin{tabular}{|c|c|c|c|c|}
\hline \multirow[t]{2}{*}{ PB } & \multirow[t]{2}{*}{ FITASE } & \multicolumn{3}{|c|}{ PARÂMETROS AVALIADOS } \\
\hline & & $\mathrm{QNC}(\mathrm{EP})^{1}$ & $\mathrm{QPC}(\mathrm{EP})^{1}$ & $\mathrm{QCaC}(\mathrm{EP})^{1}$ \\
\hline & Proteína x Fitase & $(\mathrm{P}=0,102)$ & $(\mathrm{P}=0,00)$ & $(\mathrm{P}=0,00)$ \\
\hline 16 & 0 & $3,02 \pm 0,06$ & $0,59 \pm 0,01$ & $0,62 \pm 0,01$ \\
\hline 16 & 300 & $2,97 \pm 0,06$ & $0,58 \pm 0,01$ & $0,74 \pm 0,01$ \\
\hline 16 & 600 & $3,00 \pm 0,05$ & $0,52 \pm 0,01$ & $0,65 \pm 0,01$ \\
\hline 16 & 900 & $2,96 \pm 0,05$ & $0,47 \pm 0,01$ & $0,74 \pm 0,01$ \\
\hline \multirow{3}{*}{\multicolumn{2}{|c|}{$\begin{array}{l}\text { Regressão Linear } \\
\text { Regressão Quadrática } \\
\text { Regressão Cúbica }\end{array}$}} & $(\mathrm{P}=0,56)$ & $(\mathrm{P}=0,00)$ & $(\mathrm{P}=0,001)$ \\
\hline & & $(\mathrm{P}=0,95)$ & $(\mathrm{P}=0,03)$ & $(\mathrm{P}=0,388)$ \\
\hline & & $(\mathrm{P}=0,49)$ & $(\mathrm{P}=0,119)$ & $(\mathrm{P}=0,00)$ \\
\hline 18 & 0 & $3,40 \pm 0,05$ & $0,72 \pm 0,01$ & $0,83 \pm 0,01$ \\
\hline 18 & 300 & $3,36 \pm 0,06$ & $0,59 \pm 0,01$ & $0,65 \pm 0,01$ \\
\hline 18 & 600 & $3,16 \pm 0,06$ & $0,48 \pm 0,01$ & $0,70 \pm 0,01$ \\
\hline 18 & 900 & $3,39 \pm 0,05$ & $0,49 \pm 0,01$ & $0,70 \pm 0,01$ \\
\hline \multirow{3}{*}{\multicolumn{2}{|c|}{$\begin{array}{l}\text { Regressão Linear } \\
\text { Regressão Quadrática } \\
\text { Regressão Cúbica }\end{array}$}} & $(\mathrm{P}=0,325)$ & $(\mathrm{P}=0,00)$ & $(\mathrm{P}=0,00)$ \\
\hline & & $(\mathrm{P}=0,033)$ & $(\mathrm{P}=0,00)$ & $(\mathrm{P}=0,00)$ \\
\hline & & $(\mathrm{P}=0,056)$ & $(\mathrm{P}=0,036)$ & $(\mathrm{P}=0,002)$ \\
\hline
\end{tabular}

$\mathrm{EP}=$ Erro padrão da média

1 -Houve interação proteína $\mathrm{x}$ fitase $(\mathrm{P} \leq 0,102)$

O consumo de fósforo e cálcio foram reduzidos na medida que foi adicionada fitase, sendo que à $\mathrm{QCaC}$ apresentou um platô, devido a sua concentração na dieta ter sido reduzida apenas em relação a dieta testemunha, não diferenciando entre os níveis de fitase adicionados. Porém, a QPC foi reduzida linearmente, principalmente na dieta com $16 \%$ de PB. Este fato ocorreu devido à concentração deste mineral nas dietas terem sido reduzidos proporcionalmente ao nível de fitase adicionado.

A fitase ocasionou redução $(\mathrm{P}<0,102)$ na quantidade de nitrogênio, fósforo e cálcio excretados pelos animais (Tabela 3). Foi encontrado um efeito quadrático $(\mathrm{P} \leq 0,034)$ na $\mathrm{QNET}$, efeito linear platô sobre a QPF e QCaF, e efeito linear $(\mathrm{P} \leq 0,003)$ sobre a QPU, no entanto, a QCaU não foi afetada $(\mathrm{P} \leq 0,615)$ pela ação da enzima. As equações estão representadas graficamente na figura 1, para os parâmetros relacionados ao fósforo, na figura 2, para os parâmetros relacionados ao cálcio e, na figura 3, para o parâmetro relacionados ao nitrogênio. Com isso, foi observado um nível máximo da fitase sobre a QNET, QPF e QCaF, pois não houve comportamento linear, exceto na QPU. Este fato aconteceu provavelmente devido ao aumento da relação Ca:P nas dietas com maiores níveis de fitase. Segundo QUIAN et al. (1996), a suplementação de

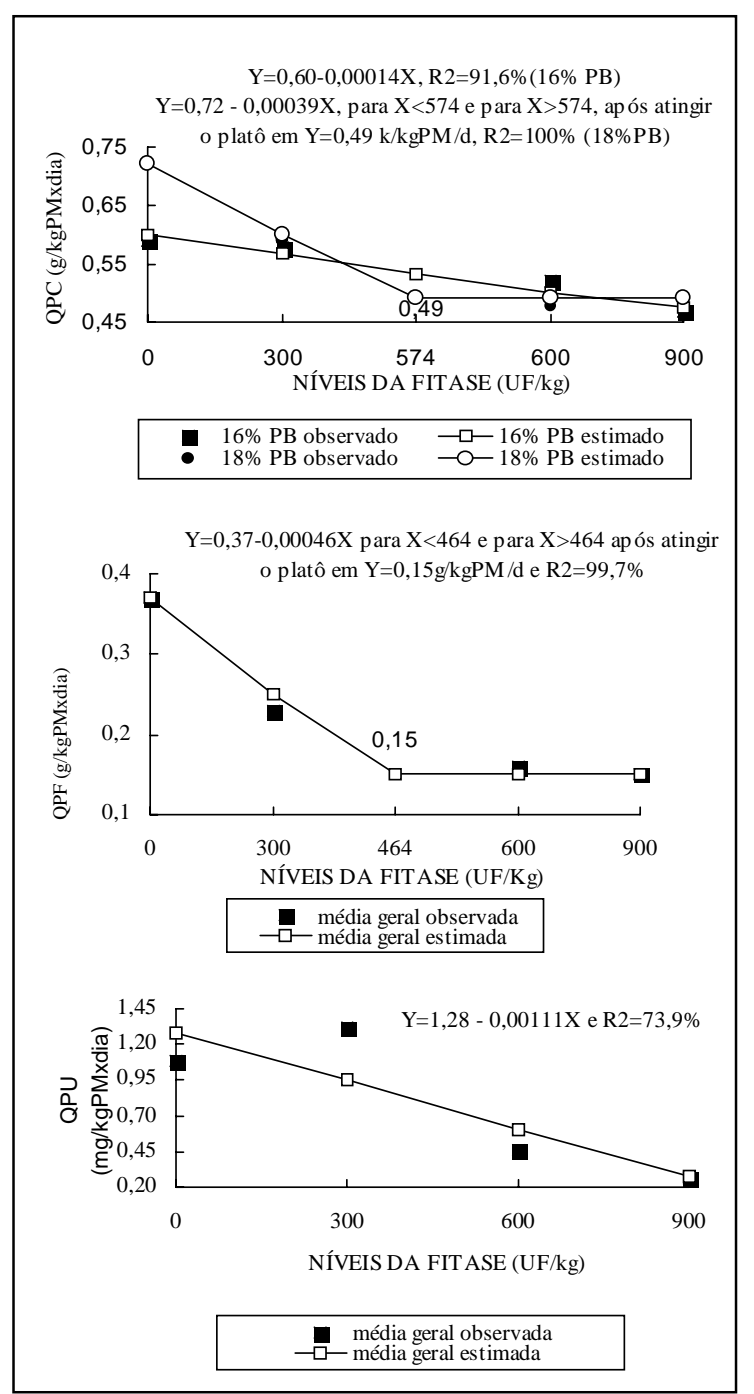

Figura 1 - Efeito dos níveis de fitase nas dietas sobre QPC $\left(\mathrm{g} / \mathrm{kg}^{0,75} \mathrm{X} \mathrm{d}\right)$ nos diferentes níveis protéicos e sobre QPF $\left(\mathrm{g} / \mathrm{kg}^{0,75} \mathrm{x} \mathrm{d}\right)$ e QPU $\left(\mathrm{mg} / \mathrm{kg}^{0,75} \mathrm{x} \mathrm{d}\right)$ independente do nível de proteína da dieta.

fitase em dietas para suínos deve ocorrer quando esta dieta apresentar uma relação $\mathrm{Ca}: \mathrm{P}$ próxima a 1,2:1. Quando a relação molar de cátions (cálcio e fósforo) estiver em 2:1 ou 3:1 com o fitato, a formação de complexo insolúvel será muito maior (OBERLEAS \& HARLAND, 1996). O presente experimento apresentou relação $\mathrm{Ca}: \mathrm{P}$ próxima a 1,2:1 nos primeiros níveis de fitase adicionado. Ao adicionar a enzima foi reduzido proporcionalmente $o$ nível de fósforo total, enquanto o teor de cálcio foi o mesmo para todos os níveis da enzima. Dessa forma, dietas que apresentaram níveis maiores de fitase tinham relação Ca:P com maior amplitude. Este resultado está de acordo com BRUCE \& SUNDST

Ciência Rural, v. 32, n. 1, 2002. 


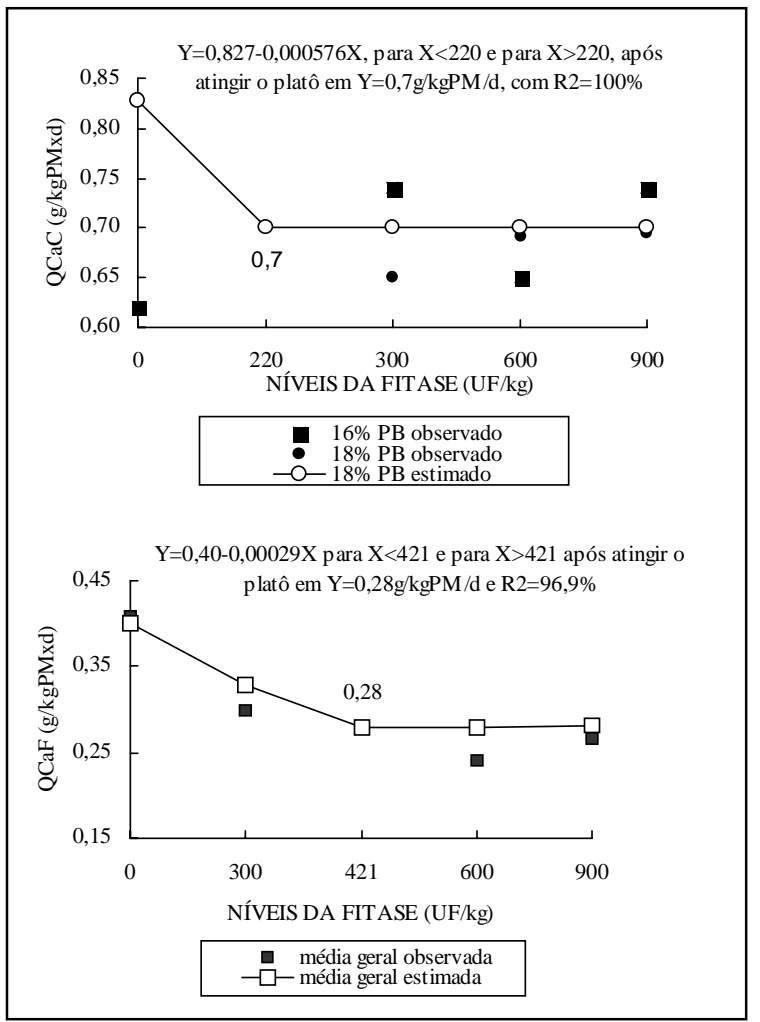

Figura 2 - Efeito dos níveis de fitase nas dietas sobre a QCaC $\left(\mathrm{g} / \mathrm{kg}^{0,75} / \mathrm{d}\right)$ e QCaF $\left(\mathrm{g} / \mathrm{kg}^{0,75} / \mathrm{d}\right)$, independente do nível protéico

(1995) que não encontraram efeito significativo sobre a retenção do nitrogênio, devido a terem mantido os mesmos níveis de cálcio nas dietas

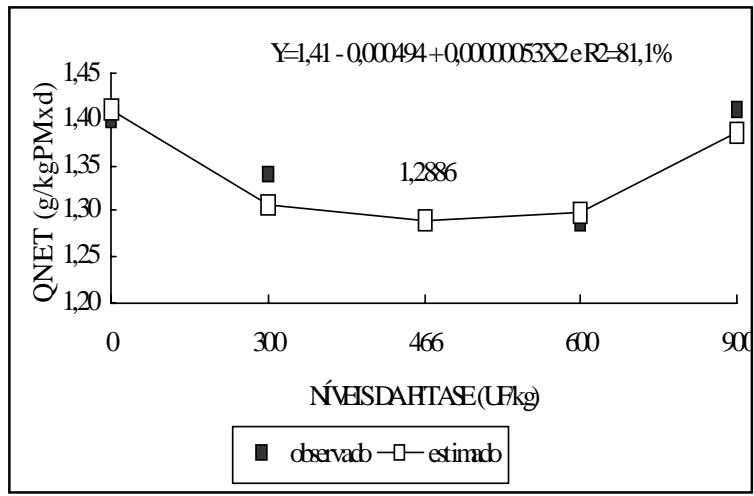

Figura 3 - Efeito dos níveis de fitase nas dietas sobre a QNET $\left(\mathrm{g} / \mathrm{kg}^{0,75} / \mathrm{d}\right)$, independente dos níveis de proteína.

suplementadas com 375 e $750 \mathrm{UF} / \mathrm{kg}$ da dieta, baixando os de fósforo proporcionalmente. Além disso, HARPER et al. (1997) encontraram uma redução na digestibilidade do fósforo em suínos em crescimento e terminação, quando alimentados com dietas onde o nível deste mineral foi reduzido, porém, ao adicionarem 250 ou $500 \mathrm{UF} / \mathrm{kg}$ nestas dietas, verificaram um aumento linear no aproveitamento do fósforo pelos animais, e a relação Ca:P utilizada nestas dietas foi no máximo de 1,3:1.

\section{CONCLUSÕES}

Níveis entre 421 - 466UF/kg da dieta são os que proporcionam redução nas quantidades de nitrogênio, fósforo e cálcio excretados pelos suínos em crescimento amenizando a carga de poluição ambiental. A eficiência da enzima em ambos os níveis de proteína é a mesma ao estudar esses parâmetros.

\section{REFERÊNCIAS BIBLIOGRÁFICAS}

AOAC (ASSOCIATION OF OFFICIAL ANALYTICAL CHEMISTS) Official methods of analysis. Arlington : AOAC, 1984. 1141p.

BASF. Sobre el tema de los aditivos para la alimentación animal: Informaciones Técnicas. Ludwigshafen: BASF, 1997, 140p.

BRUCE, J.A.M., SUNDST, F. The effect of microbial phytase in diets for pigs on apparent ileal and faecal digestibility, $\mathrm{pH}$ and

flow of digesta measurements in growing pigs fed a high-fibre diet. Can J Anim Sci, Ottawa, v.75, n.1, p.121-127, 1995.

COFFEY, M.T. An industry perspective on environmental and waste management issues: Challenge for the feed industry. In: GEORGIA NUTRITION CONFERENCE, 1992, Athens, Proceedings ... Athens : University of Georgia, 1992, p.144-148. 
CROMWELL, G.L., COFFEY, R.D., MONEGUE, H.J. et al Efficacy of low-activity, microbial phytase in improving the bioavailability of phosphorus in corn-soybean meal diets pigs. J Anim Sci, Champaign, v.73, n.2, p.449-456, 1995.

ENG, K. Nutrition, manure, environment do not equal a simple equation. Feedstuffs, Minneapolis, v.68, n.44, p.11-12, 1996.

HARPER, A.F., KORNEGAY, E.T., SCHELL, T.C. Phytase supplementation of low-phosphorus growing-finishing pig diets improves performance phosphorus digestibility and bone mineralization and reduces phosphorus excretion. J Anim Sci, Champaign, v.75, n.12, p.3174-3186, 1997.

LABTEST. Sistemas diagnósticos para determinação de fósforo inorgânico "in vitro". Belo Horizonte, 1985. (Informações do Fabricante). 4p.

LABTEST. Sistemas diagnósticos para determinação do cálcio "in vitro", Lagoa Santa, 1996. (Informações do Fabricante). 4 p.

LEHNINGER, A.L., NELSON, D.L., COX, M.M. Principles of biochemistry. 2. ed., New York: Worth, 1993. 1013p.

NRC (NUTRIENT REQUIREMENTS OF SWINE). 9. ed.
Washington, DC : National Academy, 1988, 90p.

OBERLEAS, D., HARLAND, B.F. Impact of phytate on nutrient availability. In: COELHO, M.B., KORNEGAY, E.T. (Coord.) Phytase in animal nutrition and waste management. Mount Olive : BASF Corporation, 1996. p.7784

O'DELL, B.L.; DeBOLAND, A. Complexation of phytate with proteins and cations in corn germ and oilseeds meals, J Agric Food Chem, Washington, v.24, n.4, p.804-808, 1976.

PINNOT, R. Les rejets d'azote par les élevages de porcs. Porc Magazine, Rennes, v.226, n.8, p.158-161, 1990

QUIAN, H., KORNEGAY, E.T., CONNER Jr., D.E. Adverse effects of wide calcium : phosphorus ratios on supplemental phytase. Efficacy for wealing pigs fed two dietary phosphorus levels. J Anim Sci, Champaign, v.74, n.6, p.1288-1297, 1996.

SAS. Institute (Cary, NC) version 6.4: statistic, Cary, v.2, 1990, $1686 \mathrm{p}$.

STILLBORN, H. Nutrition influences animal waste output. Feedstuffs, Minneapolis, n.4, p.20-47, 1998.

Ciência Rural, v. 32, n. 1, 2002. 